\title{
Focused section on advanced robotic systems for industrial automation
}

\author{
Huan Zhao ${ }^{1} \cdot$ LiMin Zhu $^{2} \cdot$ Yunyi Jia ${ }^{3} \cdot$ Naoyuki Kubota $^{4}$
}

Published online: 1 June 2020

(c) Springer Nature Singapore Pte Ltd. 2020

Driven by the sustained demand for increasing productivity and improving product quality, robotic systems are dramatically increasing in industrial automation and striving to appear in more and more scenarios. Advances on robotic systems for industrial automation are rapidly expanding in both the depth and extent. Better operational efficiency, accuracy, and reliability are always the endless pursuit of a robotic system. High intelligence and autonomy are imperative for robotic systems to perform increasingly complex tasks and explore wider and varying environments. This focused section is dedicated to soliciting the state-of-theart contributions addressing the fundamental challenges and practical issues in all aspects of robotic systems for industrial automation.

To disseminate current advances and gain an insight into the challenges and opportunities in this domain, this "Focused section on Advanced Robotic Systems for Industrial Automation" of the International Journal of Intelligent Robotics and Applications highlights recent efforts and important achievements in advanced Robotic Systems for

LiMin Zhu

zhulm@sjtu.edu.cn

Huan Zhao

huanzhao@hust.edu.cn

Yunyi Jia

yunyij@clemson.edu

Naoyuki Kubota

naokubota@nifty.com

1 State Key Laboratory of Digital Manufacturing Equipment and Technology, Huazhong University of Science and Technology, Wuhan 430074, China

2 State Key Laboratory of Mechanical System and Vibration, School of Mechanical Engineering, Shanghai Jiao Tong University, Shanghai 200240, China

3 Collaborative Robotics and Automation (CRA) Lab, Clemson University, Clemson, SC 29634, USA

4 Tokyo Metropolitan University, Tokyo 192-0397, Japan
Industrial Automation, including designs, planning, control, perception and learning of advanced robotic systems across industrial fields. The accepted contributions can be divided into the following three parts:

The first part contains three papers that focus on robot design, planning and control. The three papers in this part shoot a glance at the state-of-the-art advances on robot design, manipulation planning, and motion control, which endeavor to improve the efficiency, precision, robustness in robotic automation. The robot design has always been an essential issue for improving the working performance of a robot. The paper "Worst case identification-based topology optimization of a 2-DoF hybrid robotic arm" from Chong et al. presents an efficient approach to accomplish the structural topology optimization for a 2-DOF hybrid robotics arm with global validity while considering the time-varying configurations and joint forces in the whole workspace. To ensure global validity, all worst cases in aspects of kinematic characteristics and compliance are identified in the whole workpiece and taken into consideration for constructing the objective function of topology optimization. The proposed topology optimization can be solved with fast convergence by adopting a modified guide-weight method. The performance comparison between the optimized robotic arm and the baseline one shows that the optimized structure of the hybrid robotic arm has better performance on stiffness, inertia, and dynamics. For specific tasks, motion planning and compliant control are inescapable for a robot interacting with objects and environments. The paper "Integrating combined task and motion planning with compliant control" by Chen et al. introduces an integral motion planning and control method to avoid the failure of dual-arm motion for peg-in-hole assembly, especially due to the inevitable errors in the grasps of a robotic hand and absolute position errors in serial robotic manipulators. The combined task and motion planning are integrated to generate grasps and motions for a dual-arm robot to pick up objects and move them to assembly poses. The compliant strategies are used to overcome the 
calibration errors and grasp uncertainty for insertion motion. Experimental results validate that the proposed method is applicable to various peg-in-hole tasks. The paper "Neural networks-based model predictive control for precision motion tracking of a micropositioning system" by Yan et al. proposes to use a neural networks (NN) model for optimizing the parameters of the model predictive control (MPC) in a micropositioning system. The presented NN-MPC control strategy enables the precision motion tracking of a micropositioning system to be more intelligent and adaptive while comparing with conventional methods. Extensive simulation studies demonstrate the NN-MPC scheme has good robustness in the condition of varying model parameters and lots of noise.

The second part contains two papers that focus on robotic perception. The two papers in this part introduce the robotic perception of the working environment, which couples with the goal to enable the robot to interact with the environment. A simple perception environment for robot operations is the static indoor environment. The paper "An environment state perception method based on knowledge representation in dual-arm robot assembly tasks" from Wang et al. presents an environment state perception method for assembling randomly placed components by using a dual-arm robot. The paper intends to construct a global environment state model of indoor assembly scenes, which enables robots to plan continuous assembly behaviors in the built environment. By combining visual perception, point cloud process, and knowledge representation, the information of target objects is not only to be recognized but also used as part of the constructed environment for planning dual-arm robot assembly. Simulations and experimental results illustrate that the proposed approach can achieve very good environment perception performance. In many automation scenarios, a moving target is more often to be found but very challenged for robotic manipulation. The paper "Auto-Correct-Integrated trackers with and without memory of first frames" by Sekhavati and Eghbal proposes to combine different trackers for visual short-term tracking in any sequence of images, which may have lots of pose variations, target deformations and different types of occlusion. The combined trackers can minimize their faults by continuously correcting each other's faults. By testing through a database containing 7355 frames with different targets to track in different areas, experimental results show that the proposed method outperforms many of the state-of-the-art trackers as well as their components when they work independently.

The third part contains three papers that focus on robot learning. The three papers in this part show that robots could acquire good adaptability to execute flexible tasks and have strong interactivity with humans through the way of learning. Learning peg-in-hole assembly skills is still a challenge for robots because of the difficulties in collecting massive transition data and transferring skills to new tasks. The paper "Model accelerated reinforcement learning for high precision robotic assembly" from Zhao et al. presents a model accelerated reinforcement learning method to efficiently learn the assembly policy. In this paper, the assembly policy is learned with the maximum entropy reinforcement learning framework and executed with an impedance controller, which ensures exploration efficiency and allows transferring skills between tasks. To reduce sample complexity and improve training efficiency, the paper learns the environment dynamics with Gaussian Process during policy training. The learned dynamic model is utilized to improve target value estimation and generate virtual data to argument transition samples. Experimental results demonstrate that the proposed method improves the training efficiency by $31 \%$ compared with the method without model acceleration and the learned skills can be transferred to new tasks to accelerate the training for new policies. Reinforcement learning can also be used to learn robot manipulation skills. The paper "A Sample Efficient Model-based Deep Reinforcement Learning Algorithm with Experience Replay for Robot Manipulation" by Zhang et al. presents a model-based deep reinforcement learning algorithm for robotic manipulation. In this paper, a deep neural network model is utilized to simulate the system dynamics. The model is robust enough to deal with complex control tasks and possesses the generalization ability. To solve the sparse reward problem and improve the sample efficiency, this paper adopts a curiosity-based experience replay method. Simulations show great effectiveness of the proposed method. Various manipulation tasks are achieved successfully in such a complex dynamic system and the sample efficiency gets improved even in a sparse reward environment, as the learning time gets reduced considerably. Learning the human's desired trajectory or motion intention is a critical yet typical problem in physical human-robot interaction. The paper "Iterative Learning of Human Partner's Desired Trajectory for Proactive Human-Robot Collaboration" by Xia et al. presents a period-varying iterative learning control scheme for a robotic manipulator to learn a target trajectory that is planned by a human partner but unknown to the robot. The proposed method updates the robot's reference trajectory in an iterative learning manner to minimize the interaction force applied by the human. Experimental results show that the proposed method has the potential to reduce human effort in human-robot collaboration and transfer human skills to robots, which can be applied to applications such as heavy load transport in the automotive industry and compliant machining.

Together, we believe the above papers provide a useful insight into developments in the field of advanced robotic systems for industrial automation, addressing issues of robot design, planning, control, perception, and learning. We thank the editor-in-chief and staff of the International 
Journal of Intelligent Robotics and Applications for this opportunity to focus on activities in this field. This section will continue to introduce the latest research progress of advanced robot systems in the future, and keep tracking the new designs, new algorithms, theories and methods for modeling, analysis, perception, planning, implementation and/or control of advanced robot systems across industrial fields.

Publisher's Note Springer Nature remains neutral with regard to jurisdictional claims in published maps and institutional affiliations.

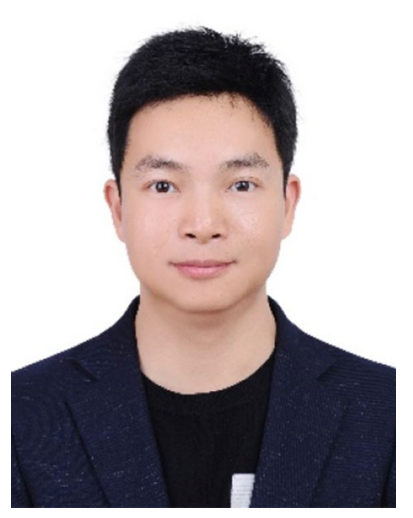

Huan Zhao received his B.E. degree from School of Mechanical Science and Engineering, Jilin University, Changchun, China, in 2006, and Ph.D. degree from School of Mechanical Engineering, Shanghai Jiao Tong University, Shanghai, China, in 2013. During 2013-2015, he was a Postdoctoral Researcher with the Huazhong University of Science and Technology. Since 2015, he has been with Huazhong University of Science and Technology. He is now an associate professor at Huazhong University of Science and Technology. He won Silver Award of Shangyin excellent mechanical doctoral thesis in 2014, excellent doctoral thesis of Shanghai in 2015, first prize of Jiangsu Science and Technology Progress Award in 2018. His research interests include force control, visual servoing and machine learning with applications to robotic machining.

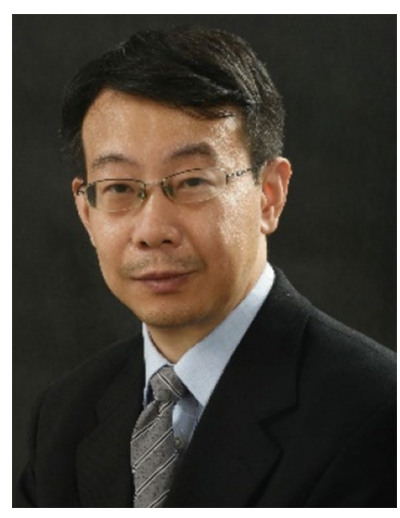

LiMin Zhu received the B.E. degree (with honors) and the Ph.D. degree both in mechanical engineering from Southeast University in 1994 and 1999, respectively. From Nov. 1999 to Jan. 2002 , he worked as a postdoctoral fellow in Huazhong University of Science and Technology. Since March 2002, he has been with Shanghai Jiao Tong University, where he is currently the "Cheung Kong" Chair Professor, head of the Department of Mechanical Engineering, vice director of the State Key Laboratory of Mechanical System and Vibration, and director of the Shanghai Key Laboratory of Networked Manufacturing and Enterprise Information. He has held the visitorship in Monash University (from Sep. 1997 to May 1998) and The City University of Hong Kong (from Dec. 2000 to Mar. 2001). His research interests include (1) Multi-axis CNC machining technology and machine tool, (2) intelligent robotic manufacturing, (3) coordinate metrology and measurement, and (4) control, sensing and instrumentation for micro/nano manufacturing. He has published 1 monograph and more than 180 international journal papers. Prof. Zhu was the recipient of the National Science Fund for Distinguished Young Scholars in 2013 and selected into the National High-level Personnel of Special Support Program in 2016. He has been an Associate Editor for the IEEE Transactions on Automation Science and Engineering. He is now a Technical Editor for the IEEE/ASME Transactions on Mechatronics, and Editorial Board Members of the International Journal of Intelligent Robotics and Applications, Proceedings of the Institution of Mechanical Engineer (IMechE), Part B: Journal of Engineering Manufacture, and Chinese Journal of Mechanical Engineering.

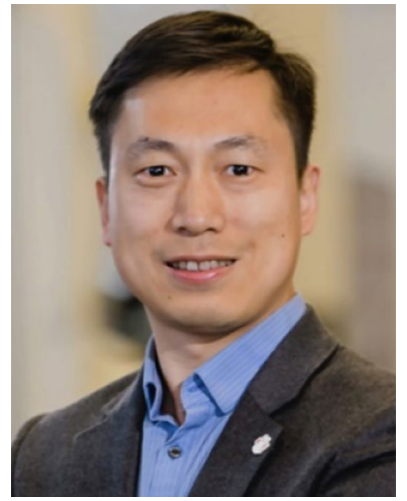

Yunyi Jia received his Ph.D. in Electrical Engineering from Michigan State University in 2014, M.S. in Control Theory and Control Engineering from South China University of Technology in 2008, and B.S. in Automation from National University of Defense Technology in 2005. He joined the Department of Automotive Engineering in 2016 as an assistant professor in the Clemson University International Center for Automotive Research (CU-ICAR). He directs the Collaborative Robotics and Automation (CRA) Lab which is located at both CU-ICAR and Center for Manufacturing Innovation (CMI). His research focuses on collaborative robotics, autonomous vehicles, and advanced sensing systems which are being supported by both federal agencies and industries. He is the recipient of the SAE Ralph R. Teetor Educational Award (2020), NSF Faculty Early Career Development (CAREER) Award (2019), NSF Computer and Information Science and Engineering (CISE) Research Initiation Initiative (CRII) Award (2018), and SAE Trevor O. Jones Outstanding Paper Award (2017).

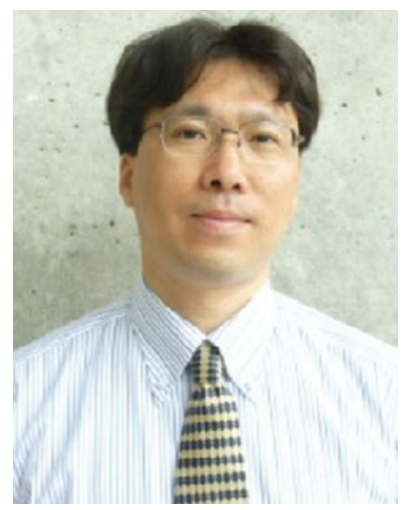

Naoyuki Kubota received the B.Sc. degree from Osaka Kyoiku University, Kashiwara, Japan, in 1992, the M.E. degree from Hokkaido University, Hokkaido, Japan, in 1994, and the D.E. degree from Nagoya University, Nagoya, Japan, in 1997. Hejoined the Osaka Institute of Technology, Osaka, Japan, in 1997. In 2000, he joined the Department of Human and Artificial Intelligence Systems, Fukui University, as an Associate Professor. He joined the Department of Mechanical Engineering, Tokyo Metropolitan University, in 2004. He is a Professor with the Department of System Design, Tokyo Metropolitan University, Tokyo, Japan. His current interests are in the fields of cognitive robotics, robot partners, coevolutionary computation, fuzzy control, spiking neural networks, and informationally structured space. 